\title{
High energy neutrinos from cosmic ray interactions in the Sun
}

\author{
Joakim Edsjö ${ }^{a}$, Jessica Elevant ${ }^{a}$, Rikard Enberg ${ }^{b}$ and Carl Niblaeus ${ }^{* a}$ \\ ${ }^{a}$ Oskar Klein Centre for Cosmoparticle Physics, Department of Physics, Stockholm University, \\ SE-10691 Stockholm, Sweden \\ ${ }^{b}$ Department of Physics and Astronomy, Uppsala University, Box 516, SE-75120 Uppsala, \\ Sweden \\ E-mail: edsjo@fysik.su.se, jessica.elevant@fysik.su.se, \\ rikard.enberg@physics.uu.se, carl.niblaeus@fysik.su.se
}

\begin{abstract}
Cosmic rays hitting the outer parts of the Sun result in showers of high energy particles. The shower particles propagate through the solar atmosphere and interact further or decay. Among the shower particles are high energy neutrinos, after production these oscillate between flavours and interact with the solar material while propagating out of the Sun to the Earth. The result is a high energy neutrino flux at the Earth that may be detectable by modern neutrino detectors such as IceCube. Such a neutrino flux will furthermore act as a background in searches for neutrinos coming from annihilations of weakly interacting massive particles, often suggested to be the dark matter in the Universe. We perform an updated calculation of the solar atmospheric neutrino flux using the code MCEq for the cascade evolution in the solar atmosphere and WimpSim for the propagation of the neutrinos from the Sun to the detector on Earth, including full three-flavour treatment of neutrino oscillations and interactions in the Sun.
\end{abstract}

35th International Cosmic Ray Conference - ICRC2017

10-20 July, 2017

Bexco, Busan, Korea

${ }^{*}$ Speaker. 


\section{Introduction}

When cosmic rays (CRs) hit particles in the Sun's atmosphere this results in cascades consisting of particles that continue to interact and decay as they travel further in the atmosphere. In the cascades neutrinos are generated mainly through the decays of pions, kaons and muons. This is described by the cascade equations, a set of coupled differential equations tracking the fluxes of the particles in the cascades as function of depth in the atmosphere. The neutrinos can propagate to Earth, leading to a high energy flux of solar atmospheric neutrinos (SAv) at Earth. The mechanism is similar to how Earth atmospheric neutrinos (EAv) are produced by CR interactions in the Earth's atmosphere and one could naively expect the SA $v$ and EA $v$ fluxes to be the same. However, an important difference is that the density in the solar atmosphere is lower, leading to a larger fraction of decays compared to interactions and therefore a larger neutrino flux. Another important difference is that the SA $v$ s will interact with the solar material and oscillate on the way from the production point to the Earth. This leads to an attenuation of the flux at high energies since the Sun then becomes opaque for neutrinos and a different ratio between the fluxes of different flavour due to flavour oscillations.

The SA $v$ and EA $v$ fluxes will also be different due to the solar magnetic field. In the region of interest near the solar surface the magnetic field is complicated and our knowledge to a a large extent relies on simulations and modelling [1-3]. The magnetic field will affect the SA $v$ by deflecting some CRs and preventing them from interacting and producing cascades, but it will also affect the propagation of charged particles in the cascades. The magnetic effect on the CRs will be larger for lower energy particles and for high energy particles the magnetic field can be neglected. It is unclear at what energy the magnetic field starts to become important. In Ref. [4] the neutrino energies above which magnetic effects can be neglected is estimated as $E_{v} \sim 200 \mathrm{GeV}$. Observations of the solar gamma-ray flux made by Fermi-LAT [5, 6] are higher than expected for a non-magnetic Sun [4] up to around $100 \mathrm{GeV}$, suggesting that CR primaries are affected up to $E \sim 1 \mathrm{TeV}$. The effect is further complicated by the 11-year solar cycle, resulting in a time-dependent solar magnetic field and hence gamma-ray (and in principle neutrino) flux [6]. In our study we neglect the solar magnetic field and calculate the SA $v$ flux from a non-magnetic Sun which should be accurate for high energies but presents an uncertainty at lower energies.

The SA $v$ flux has been studied before $[4,7-12]$ as well as in recent studies [13-16]. In Ref. [13] we have performed an updated calculation of the SA $v$ flux at Earth including a full threeflavour treatment of oscillations and interactions. We have used an event-based Monte Carlo code for our calculations which is released publicly [17] and allows for the calculation of neutrino or muon fluxes in a detector at Earth. Our improvements include, apart from the aforementioned interaction and oscillation treatment, for example the use of updated models for the incoming CR flux, the solar density model as well as the hadronic interaction models used. We also calculate the production fluxes at more values of the impact parameter (see Fig. 1 for a view of the geometry) to ensure small errors when the fluxes are interpolated.

The SA $v$ provides an almost irreducible background in the searches for an excess of neutrinos in the direction of the Sun coming from annihilations of weakly interacting massive particles (WIMPs), often suggested to be the dark matter in the Universe. Therefore it is crucial to properly characterise the SAv flux in order to be able to distinguish it from a WIMP-induced neutrino flux 
as well as possible.

\section{Summary of calculation}

Our calculation is divided in two parts: the calculation of the production fluxes of neutrinos in the solar atmosphere is performed with the code MCEq $[18,19]$ and the propagation of the neutrinos from production to a detector on Earth is done with an updated version of the code WimpSim [13, $17,20,21]$. MCEq is a one-dimensional cascade equation solver from which we obtain the neutrino production fluxes in the atmosphere as function of energy $E$, impact parameter $b$, flavour $\alpha$ and travelled path length $\ell$ in the atmosphere. MCEq is originally written to calculate neutrino fluxes in the Earth's atmosphere, hence we have made some modifications to be able to use it in a solar environment. We have (i) changed to a solar geometry (see Fig. 1 for a schematic view), (ii) changed to density profiles appropriate for the outer parts of the Sun, (iii) included muon energy loss and (iv) used cross sections and yields calculated for hadronic projectiles on protons rather than air.

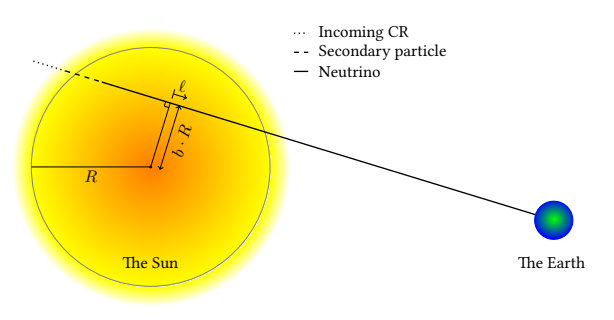

Figure 1: A schematic view of the geometry used in the calculation.

We solve the cascade equations with MCEq for 14 different values of the impact parameter $b$ between 0 and 1.002 , spaced with more values near the solar limb, since the neutrino fluxes rise quickly when $b$ approaches $b=1$. We use two different models for the incoming CR flux, the $H 3 a$ model from Ref. [22] and the GST 4-gen model from Ref. [23]. For the density profile we use two different profiles which we refer to as Ser+Stein and Ser+GS98. Both use the Serenelli SSM from Ref. [24] for the interior. For the part just below the solar surface this is combined with the density from a magneto-hydrodynamic simulation by Stein et al. [25] ${ }^{*}$ in the first case and the model by Grevesse and Sauval [26] in the second case. In both profiles we use the same profile as in Ref. [10] for the part outside the solar surface, an exponential fit to data in Ref. [27].

The production fluxes from MCEq are read into WimpSim where they are interpolated linearly in $\ell, b$ and $\log E$. We then sample events from these using acceptance-rejection sampling. The event generation results in a sample of neutrino events distributed in $E, b$ according to the production fluxes and path length of production and $\alpha$ according to the production fluxes differential in $\ell$ (so that most neutrinos are produced just below the solar surface) with weights such that the sum of all events gives the flux in units $\left(\mathrm{cm}^{-1} \mathrm{~s}\right)$ as seen from Earth. The neutrinos are then propagated from the production point first through the Sun, where they matter oscillate and interact with the solar material, and then propagated with vacuum oscillations from the Sun to a detector on Earth. At the

\footnotetext{
*As obtained from Bob Stein's webpage: http://steinr.pa.msu.edu/ bob/data.html.
} 
detector the neutrinos are allowed to interact and as output we provide either the resulting muon flux or the neutrino fluxes before interaction.

The deep inelastic interactions between neutrinos and nucleons are simulated with nusigma [28] where the CTEQ6-DIS parton distribution functions [29] are used. In the case of charged current interactions, electrons and muons are quickly stopped in the dense interior and removed from the calculation whereas tau leptons decay quickly, producing new low energy neutrinos which we include, simulating the decay with Pythia 6.4.26 [30].

Oscillations are included in a complete three-flavour framework taking into account vacuum oscillations, matter effects and interactions. Our calculations are performed with three sets of mixing parameters-no oscillations at all and the best-fit values from Ref. [31, 32] for normal and inverted mass ordering respectively. The case with no oscillations is of course unphysical and only used as a reference result. The oscillation parameter values that we use are shown in Tab. 2.

We use the same density profile for interactions, composition and electron density (relevant for matter oscillations) as we use for the solar interior in MCEq, the Serenelli SSM [24].

\begin{tabular}{crrrrrr}
\hline \hline & $\theta_{12}\left(^{\circ}\right)$ & $\theta_{23}\left(^{\circ}\right)$ & $\theta_{13}\left(^{\circ}\right)$ & $\delta_{C P}\left({ }^{\circ}\right)$ & $\Delta m_{21}^{2}\left(\mathrm{eV}^{2}\right)$ & $\Delta m_{31}^{2}\left(\mathrm{eV}^{2}\right)$ \\
\hline No osc. & 0 & 0 & 0 & 0 & $0^{\dagger}$ & $0^{\dagger}$ \\
Normal ordering & 33.56 & 41.6 & 8.46 & 261 & $7.50 \cdot 10^{-5}$ & $+2.524 \cdot 10^{-3}$ \\
Inverted ordering & 33.56 & 50.0 & 8.49 & 277 & $7.50 \cdot 10^{-5}$ & $-2.439 \cdot 10^{-3}$ \\
\hline \hline
\end{tabular}

Table 1: The three different sets of values we use for the oscillation parameters. In the case of normal and inverted mass ordering the values used are the best-fit values from Ref. [31, 32].

\section{Results}

We show in this section results for our benchmark case that uses $H 3 a$ for the CR flux, Ser+Stein for the density and normal ordering for the mass hierarchy. In Fig. 2 we show the flux at production, after passage through the Sun, at $1 \mathrm{AU}$ from the Sun and propagated to the detector. The difference between the latter two is that the last curve takes into account the eccentricity of the Earth orbit and includes the time-averaging over the distance between the Sun and the Earth. Wiggles in the curves represent neutrino oscillations. These are well approximated by only vacuum oscillations, as we have also checked by running the code with matter effects turned off. Oscillations on the way through the Sun are mainly affecting the muon and tau neutrinos as the oscillation length that is important for electron neutrino oscillations is long compared to the solar radius for the energies considered. Between the Sun and the Earth all flavours mix and at low energies, where the oscillation lengths are short compared to the travelled distance, the flavour ratio is changed from $1: 2: 0$ at production to approximately $1: 1: 1$ whereas at higher energies the oscillation lengths are sufficiently long that they can not be averaged over. Between the production fluxes and the fluxes after passage through the Sun one can see the effect of interactions, most visible for $v_{e}$. The flux is in that case clearly attenuated after passing through the Sun.

\footnotetext{
${ }^{\dagger}$ To avoid numerical issues we need to set the $\Delta m_{i j}^{2}$ to non-zero values in the code.
} 

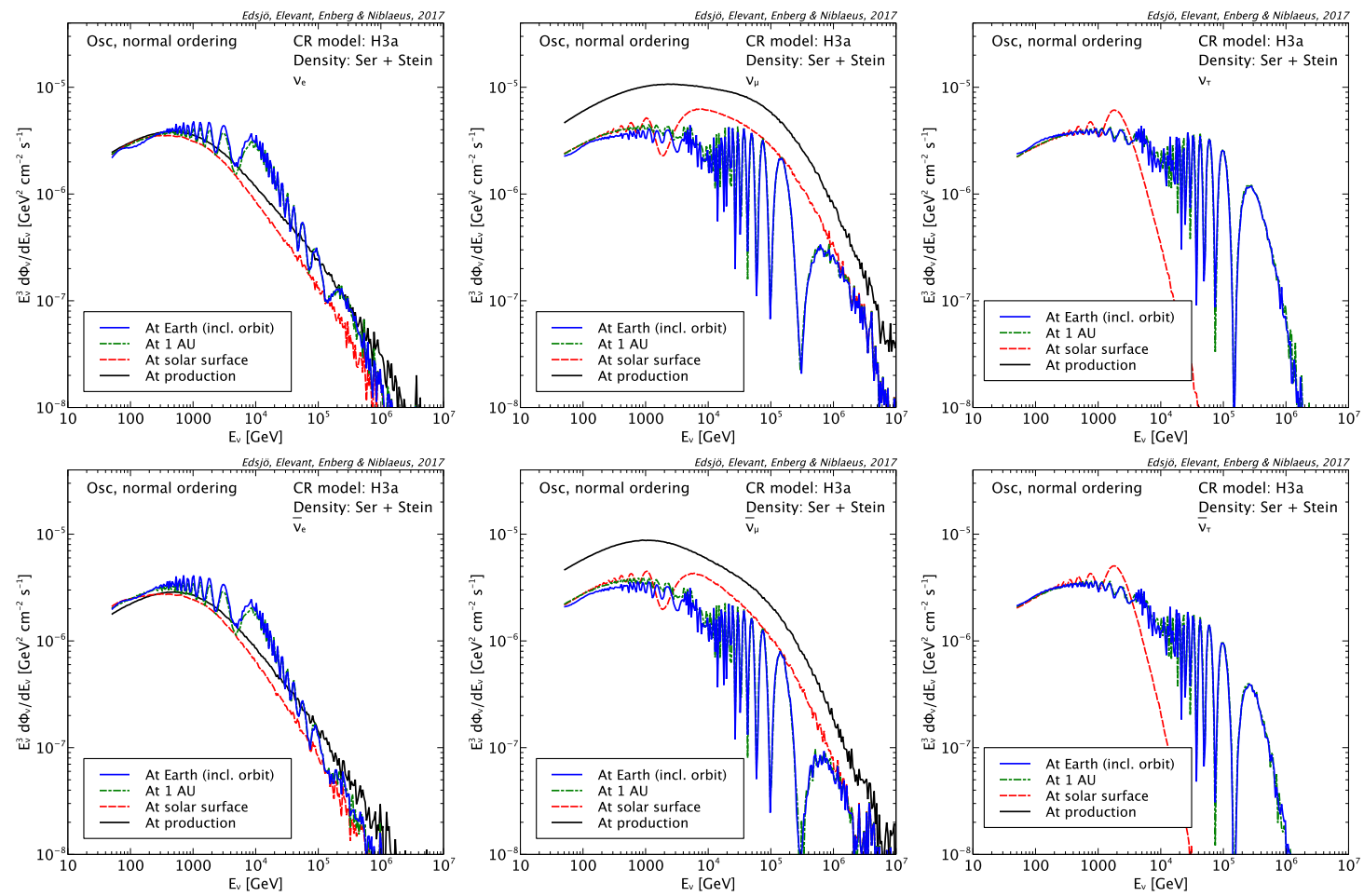

Figure 2: The fluxes of neutrinos integrated over the solar disk separated by flavour, in each plot at four different points: at production (black solid), after having passed through the Sun (red dashed), at $1 \mathrm{AU}$ from the Sun (green dot-dashed) and at the detector (blue solid). The last curve is time-averaged over the austral winter, including the eccentricity of the Earth's orbit. The flavour goes from $v_{e}$ to $v_{\mu}$ to $v_{\tau}$ from left to right and the upper three are neutrino fluxes while the lower three are anti-neutrino fluxes.

In Fig. 3 we show the flux of $v_{\mu}+\bar{v}_{\mu}$ per solid angle as function of angle from the EarthSun axis (that is directly related to the impact parameter). Especially for high energies, the flux increases as the angle approaches the solar radius at $\theta \sim 0.26^{\circ}$ where it quickly falls off. For low values of $\theta$ and high energy the neutrinos are stopped by interactions. As a reference we show the EA $v$ flux per solid angle. The EAv flux at the position of the Sun depends on where the Sun is on the sky, therefore we show the EA $v$ flux as a band between the two extremes of purely horizontal and purely vertical flux.

The SA $v$ flux will be dominated by events from large values of $b$ near the solar limb. This is due to several effects: (i) the density where the cascades are formed in the CR interactions with the atmosphere is lower at large $b$, leading to a larger fraction of meson decays compared to interactions, (ii) interactions stop neutrinos at low $b$ from passing through the Sun and (iii) from purely geometrical considerations the solid angle is larger for larger $b$. The last of these effects is not included in Fig. 3 and will, when integrating the flux over the solar disk, further enhance the dependence of large $b$ in the integrated flux.

By folding the fluxes of muon neutrinos and antineutrinos with the effective area of a neutrino telescope and integrating over energy we obtain the number of events expected from the SAv flux. In Tab. 3 we show the number of events estimated in the IceCube detector with the effective areas 

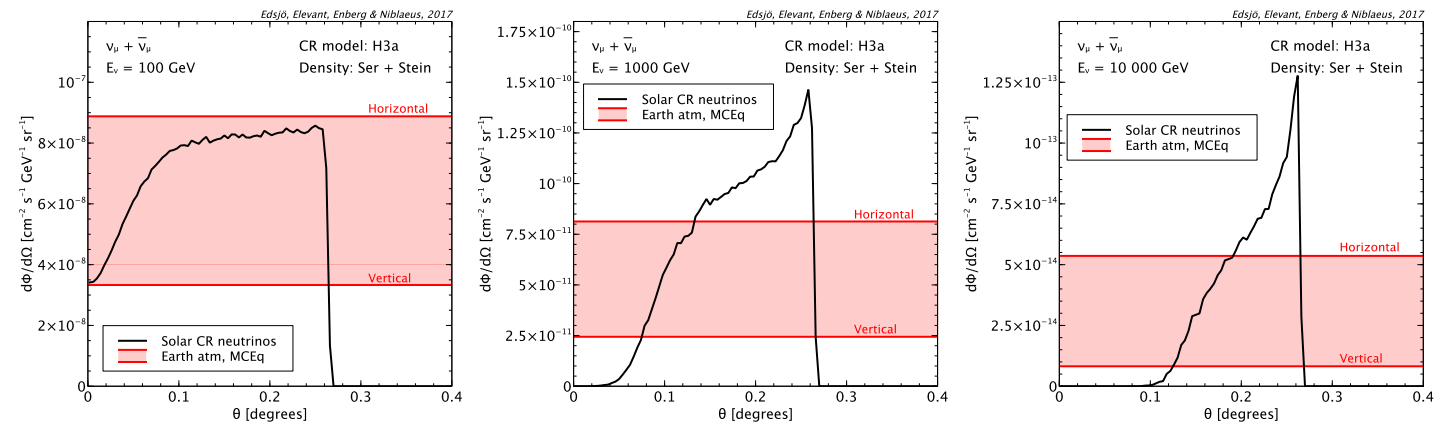

Figure 3: The flux of $v_{\mu}+\bar{v}_{\mu}$ per solid angle, comparing the SA $v$ and the EAv fluxes. The EA $v$ flux is shown as a band between the two extremes of purely horizontal and purely vertical flux. The EAv flux is calculated with MCEq.

from Ref. [33] (IC-79) and from the recent three-year analysis in Ref. [34] (IC3)

\begin{tabular}{lrr}
\hline \hline & \multicolumn{2}{c}{ Events per year } \\
\cline { 2 - 3 } Oscillation scenario & $I C-79$ & IC3 \\
\hline Normal ordering & 1.17 & 2.26 \\
Inverted ordering & 1.40 & 2.70 \\
\hline \hline
\end{tabular}

Table 2: Number of events per year of detector lifetime in IceCube. We have here calculated the number of events with the IC-79 [33] and IC3 [34] effective areas, integrating from $50 \mathrm{GeV}$ neutrino energy.

The SA $v$ flux is an almost irreducible background for the searches for neutrinos from WIMP annihilations in the Sun. In principle the angular and energy dependences are different for the two neutrino signals but present limitations in terms of energy and angular resolution mean that distinguishing them will be difficult. We can estimate a sensitivity floor for the spin-dependent WIMP-proton scattering cross section $\sigma_{\chi p}^{\mathrm{SD}}$ that neutrino telescopes use to present the limits on the WIMP capture rate in the Sun. In our analysis we estimate the sensitivity floor in the $m_{\chi}-\sigma_{\chi p}^{\mathrm{SD}}$ plane by setting the number of SA $v$ events and events from WIMP annihilations equal, i.e. we shift $\sigma_{\chi p}^{\mathrm{SD}}$ until we get as many events from both neutrino signals.

We show in Fig. 4 the result, comparing also to similar studies in Refs. [14] (FJAWs) and [15] (NBPR). Our three curves differ in how they estimate the number of events. The solid blue line integrates the flux from $50 \mathrm{GeV}$ muon energy and upwards using entering and starting events, the dashed blue line integrates from $50 \mathrm{GeV}$ muon energy and the dotted blue line from $1 \mathrm{GeV}$ muon energy, and both the latter use the IC3 effective area. In all cases we estimate the number of SA $v$ and WIMP-induced events in the same way. For the green curve from Ref. [14] the number of events is calculated in a different way, assuming some distinction between SA $v$ and WIMP-induced events and thereby restricting the energy integration range, resulting in a lower floor. We caution that the curve for the sensitivity floor is greatly dependent on the specifics of how we estimate the

\footnotetext{
${ }^{\ddagger}$ We use the highest effective area at a given energy in both cases, i.e. the DeepCore (IceCube) selection at low (high) energy for IC3 and the highest of the SL, WL and WH criteria at every energy for IC-79.
} 
number of events, especially for lower WIMP masses where very few events are expected due to the small effective area.
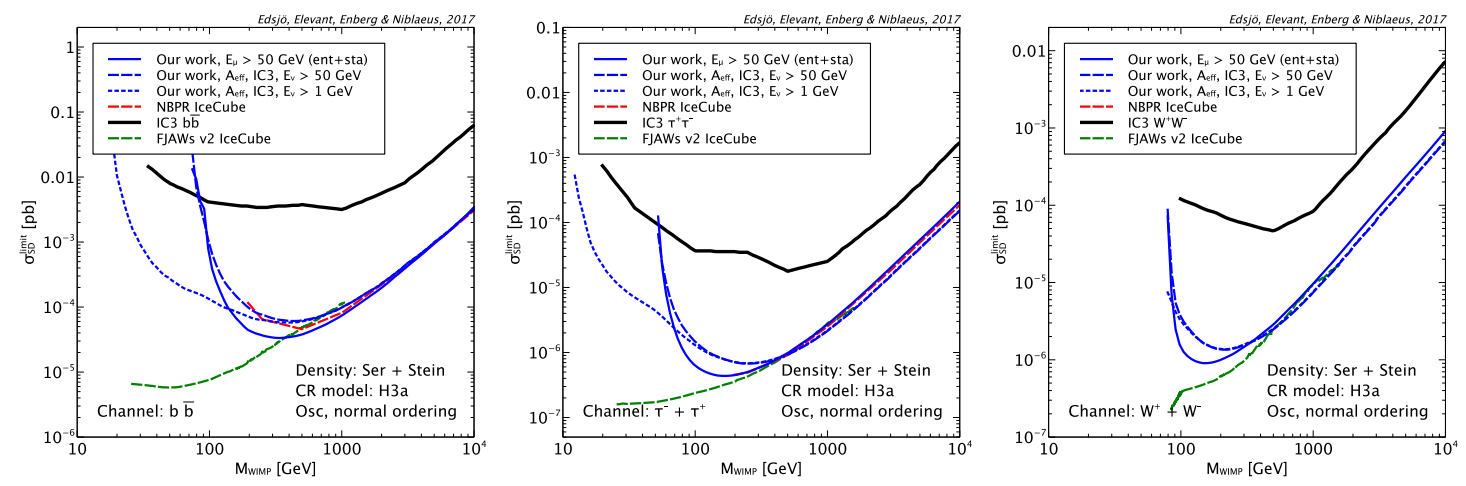

Figure 4: The sensitivity floor obtained as described in the main text. Our three results calculate the number of events in three different ways. In the first (solid) the muon flux is integrated from a threshold of $E_{\mu}=$ $50 \mathrm{GeV}$, including entering and starting events in the detector whereas in the latter two the IC 3 effective area for $v_{\mu}$ and $\bar{v}_{\mu}$ is used, integrating from $E_{\mu}=50 \mathrm{GeV}$ (dashed) and $E_{\mu}=50 \mathrm{GeV}$ (dotted) respectively. The red and green dashed lines are similar estimates made in Refs. [14] (FJAWs) and [15] (NBPR). The black solid line is the recent IceCube limit on the spin-dependent WIMP-proton cross section [34].

\section{Summary and conclusions}

We have made a calculation of the flux of solar atmospheric neutrinos produced in cosmic ray interactions in the outer parts of the sun. We have used MCEq for the calculation of the neutrino fluxes in the solar atmosphere and the event-based package WimpSim for the propagation of neutrinos from production to the detector. The results indicate around 1-3 SAv events per year in an IceCube-like detector. The SA $v$ flux is a background for the searches for neutrinos from WIMP annihilations in the solar core that is currently not included in experimental analyses. Our results for the sensitivity floor that the SA $v$ flux presents for the limits on the WIMP-proton spin-dependent cross section indicates that current neutrino telescopes do not probe cross sections where the number of SA $v$ events are comparable to that expected from WIMP annihilations but the SA $v$ flux can become a difficult background in the future, and if a small number of high energy neutrinos from the Sun are detected, a distinction may in any case be tough due to the limiting energy and angular resolutions available.

\section{Outlook}

We have not included effects of the solar magnetic field in our analysis. This introduces an uncertainty especially at low energies. Future studies should try to estimate the magnitude of this uncertainty and what the effects of the magnetic field can be for the SA $v$ flux. A proper detector simulation and detector analysis should also be made to get a more certain estimate of the sensitivity floor for solar WIMP searches and how well a WIMP-induced neutrino flux and the SA $v$ flux can be distinguished. 


\section{References}

[1] T. Wiegelmann, J. K. Thalmann, and S. K. Solanki, The Astronomy and Astrophysics Review 22.1 (2014), p. 78, ISSN: 1432-0754.

[2] D. H. Mackay and A. R. Yeates, Living Rev. Sol. Phys. 9 (2012), p. 6, arXiv: 1211.6545 [astro-ph. SR].

[3] P. Riley et al., The Astrophysical Journal 653.2 (2006), p. 1510.

[4] D. Seckel, T. Stanev, and T. K. Gaisser, Astrophys. J. 382 (1991), pp. 652-666.

[5] A. A. Abdo et al., Astrophys. J. 734 (2011), p. 116, arXiv: 1104.2093 [astro-ph. HE] .

[6] K. C. Y. Ng, J. F. Beacom, A. H. G. Peter, and C. Rott, Phys. Rev. D94.2 (2016), p. 023004, arXiv: 1508.06276 [astro-ph.HE].

[7] I. V. Moskalenko, S. Karakula, and W. Tkaczyk, 22nd International Cosmic Ray Conference (ICRC 1991) Dublin, Ireland, August 11-23, 1991, 1991, pp. 638-641.

[8] I. V. Moskalenko, S. Karakula, and W. Tkaczyk, Astron. Astrophys. 248 (1991), pp. L5-L6.

[9] I. V. Moskalenko and S. Karakula, J. Phys. G19 (1993), pp. 1399-1406.

[10] G. Ingelman and M. Thunman, Phys. Rev. D54 (1996), pp. 4385-4392, arXiv: 9604288 [ hep-ph] .

[11] C. Hettlage, K. Mannheim, and J. G. Learned, Astropart. Phys. 13 (2000), pp. 45-50, arXiv: 9910208 [astro-ph].

[12] G. L. Fogli et al., Phys. Rev. D74 (2006), p. 093004, arXiv: 0608321 [hep-ph ] .

[13] J. Edsjö, J. Elevant, R. Enberg, and C. Niblaeus, JCAP 2017.06 (2017), p. 033, arXiv: 1704.02892 [astro-ph. HE].

[14] C. A. Argüelles, G. de Wasseige, A. Fedynitch, and B. J. P. Jones (2017), arXiv: 1703.07798 [astro-ph. HE].

[15] K. C. Y. Ng, J. F. Beacom, A. H. G. Peter, and C. Rott (2017), arXiv: 1703.10280 [astro-ph. HE] .

[16] M. Masip (2017), arXiv: 1706.01290 [hep-ph ].

[17] J. Edsjö, J. Elevant, and C. Niblaeus, http://www. fysik.su.se/ eds jo/wimpsim/, 2017.

[18] A Fedynitch et al., https://github.com/afedynitch/MCEq, 2017.

[19] A. Fedynitch et al., EPJ Web Conf. 99 (2015), p. 08001, arXiv: 1503.00544 [hep-ph] .

[20] M. Blennow, J. Edsjö, and T. Ohlsson, JCAP 0801 (2008), p. 021, arXiv: 0709.3898 [hep-ph].

[21] J. Edsjö, http://www. fysik.su.se/ edsjo/wimpsim/, 2007.

[22] T. K. Gaisser, Astropart. Phys. 35 (2012), pp. 801-806, arXiv: 1111.6675 [astro-ph. HE] .

[23] T. K. Gaisser, T. Stanev, and S. Tilav, Front. Phys.(Beijing) 8 (2013), pp. 748-758, arXiv: 1303. 3565 [astro-ph. HE].

[24] A. Serenelli, S. Basu, J. W. Ferguson, and M. Asplund, Astrophys. J. 705 (2009), pp. L123-L127, arXiv: 0909.2668 [astro-ph.SR].

[25] R. F. Stein and Å. Nordlund, Ap. J. 499 (May 1998), pp. 914-933.

[26] N. Grevesse and A. J. Sauval, Space Sci. Rev. 85 (1998), pp. 161-174.

[27] J. E. Vernazza, E. H. Avrett, and R. Loeser, The Astrophysical Journal Supplement Series 45 (1981), p. 635, ISSN: 0067-0049.

[28] J. Edsjö, http://www.fysik.su.se/ edsjo/wimpsim/, 2007.

[29] S. Kretzer, H. L. Lai, F. I. Olness, and W. K. Tung, Phys. Rev. D69 (2004), p. 114005, arXiv: $0307022[$ hep-ph].

[30] T. Sjöstrand, S. Mrenna, and P. Z. Skands, JHEP 05 (2006), p. 026, arXiv: 0603175 [hep-ph] .

[31] I. Esteban et al. (2016), arXiv: 1611.01514 [hep-ph] .

[32] I. Esteban et al., http://www.nu-fit.org/, 2016.

[33] M. G. Aartsen et al., JCAP 1604.04 (2016), p. 022, arXiv: 1601.00653 [ hep-ph ].

[34] M. G. Aartsen et al., Eur. Phys. J. C77.3 (2017), p. 146, arXiv: 1612.05949 [astro-ph. HE]. 